\title{
Inhibition of CD73 stimulates the migration and invasion of B16F10 melanoma cells in vitro, but results in impaired angiogenesis and reduced melanoma growth in vivo
}

\author{
PATRYCJA KOSZAŁKA, ANNA PRYSZLAK, MONIKA GOŁUŃSKA, JUSTYNA KOLASA, \\ GRZEGORZ STASIŁOJĆ, ANDRZEJ C. SKŁADANOWSKI and JACEK J. BIGDA \\ Department of Medical Biotechnology, Intercollegiate Faculty of Biotechnology UG-MUG, \\ Medical University of Gdańsk, 80-211 Gdańsk, Poland
}

Received September 30, 2013; Accepted October 29, 2013

DOI: $10.3892 /$ or.2013.2883

\begin{abstract}
The role of ecto-5'-nucleotidase (CD73), an enzyme providing interstitial adenosine, was investigated in B16F10 melanoma progression. Chemical inhibition of CD73 decreased adherence of cells to extracellular matrix proteins in vitro and led to enhanced migration and invasion. Both processes were reversed by adenosine receptor agonists. In CD73-deficient mice, tumor growth was decreased in comparison with that of wild-type animals. Additionally, the vasculature of CD73-inhibited tumors was impaired and neoangiogenesis in Matrigel plugs was reduced. It is, therefore, proposed that although CD73 shows anti-invasive and antimigratory function in B16F10 melanoma cells, its proangiogenic action is prevalent in vivo and may contribute to increased tumor growth.
\end{abstract}

\section{Introduction}

Ecto-5'-nucleotidase (CD73, eN) is a glycosylphosphatidylinositol-linked sialylglycoprotein anchored to the outer surface of the plasma membrane and is expressed in a large number of cell types. CD73 is able to hydrolyze extracellular nucleoside monophosphates to nucleosides. As it is highly specific for 5'-AMP, it can generate bioactive adenosine during the end stage of the ectonucleotide breakdown cascade where, along with CD39 (nucleoside triphosphate diphosphohydrolase-1; NTPDase1), the sequential hydrolysis of ATP to AMP occurs $(1,2)$. According to the hypoxic hypothesis, such nucleotide breakdown and adenosine accumulation occur in solid tumors with no adequate blood supply $(3,4)$. Hypoxic conditions can stimulate the process of tumor angiogenesis via direct

Correspondence to: Dr Patrycja Koszałka, Division of Cell Biology, Department of Medical Biotechnology, Intercollegiate Faculty of Biotechnology UG-MUG, Medical University of Gdańsk, ul. Dębinki 1, 80-211 Gdańsk, Poland

E-mail:pkosz@gumed.edu.pl

Key words: melanoma, ecto-5'-nucleotidase, adenosine 5'- $\alpha, \beta$-methylene diphosphate, angiogenesis mitogenic effects on vasculature and the production of proangiogenic factors $(5,6)$.

Adenosine can signal via any of four membrane G-coupled receptors (ARs): $A_{1}, A_{2 A}, A_{2 B}$ and $A_{3}$. Each of these ARs functions via a different internal effector mechanism and exhibits a distinct pattern of tissue distribution. Different ARs were found to be upregulated in cancer cells (7). To date, there is no universal hypothesis regarding the role of different ARs in tumor growth. For instance, activation of the $A_{1} A R$ can impair glioblastoma growth, but it enhances breast cancer cell proliferation $(4,7,8) . \mathrm{A}_{2 \mathrm{~A}} \mathrm{AR}$ is key in mediating the adenosine-induced anti-inflammatory response to tumor cells via inhibition of T-cell function, which leads to a decrease in the proliferation of hormone-dependent breast cancer cells, but paradoxically leads to an increase in the viability of human melanoma cells $(4,7,9)$. Stimulation of $\mathrm{A}_{3} \mathrm{AR}$ in normal cells can induce the production of growth factors; however, in tumor cells, $\mathrm{A}_{3} \mathrm{AR}$ expression is frequently upregulated and apoptosis is induced or growth is inhibited (4,7). Finally, adenosine receptors influence the development of the tumor vascular bed through inhibition of endothelial cell growth via $A_{1} A R$ or stimulation of angiogenesis through both $A_{2 A} A R$ and $A_{2 B} A R(7,8)$.

Evidence that CD73 plays a direct role in tumor progression via regulation of cell adhesion and T-cell signaling was outlined by several investigators (10-12). Other mechanisms were also proposed; for example, CD73 interacts with tenascin$\mathrm{C}$ and leads to CD73 inhibition, which results in a decrease in extracellular adenosine and weakly interferes with the anti-adhesive properties of tenascin- $\mathrm{C}$ in the context of other ECM proteins $(13,14)$. The indication of a crucial function for CD73 in the regulation of the invasive potential of tumor cells is frequently raised.

Recently, it was reported that both CD73-deficiency in transgenic mice and pharmacological inhibition of CD73 in wild-type mice significantly slows the development of subcutaneous tumors, experimental lung metastases $(9,15,16)$ and human xenograft tumors $(17,18)$. CD73 was further suggested to be a potential negative prognostic marker in certain cancer types, such as human colorectal cancer (19). CD73 overexpression in some human breast cancer cell lines increased their ability to invade, migrate and adhere to ECM proteins, thus 
increasing their metastatic potential. Furthermore, inhibition of CD73 activity reversed those effects $(18,20)$. Nonetheless, some clinical studies have shown that CD73 exhibits low activity in breast cancer cells when compared to the adjacent non-involved tissue $(21,22)$ and that elevated CD73 expression in tumor cells was a positive prognostic marker for disease-free survival in stage I-III breast cancer patients (23). Therefore, the precise involvement of CD73 in tumor progression requires further clarification.

The murine B16F10 melanoma cell line is frequently used as the model of choice for studies regarding tumor progression as it is highly metastatic and syngenic with the C57BL/6 mouse strain which is commonly used for transgenesis. It has been previously reported that the B16F10 cell line shows low, although significant, activity of ecto-5'-nucleotidase (CD73). Specifically, CD73 activity was 50\% lower in the B16F10 line when compared to two low-metastatic variants: B16-F1 and B16-F10 ${ }^{\mathrm{Lr}}$ (lymphocyte resistant) $(24,25)$.

In the present study, we analyzed the influence of CD73 activity on B16F10 cell migration and invasion potential in vitro, its effects on subcutaneous tumor growth and the development of the vascular bed in CD73-deficient mice.

\section{Materials and methods}

Reagents. Anti-CD73 mAb (clone 2B6), anti-CD39 mAb (clone H-85), HRP-conjugated mAb and A375, MDA-MB-231 and HEK293T whole cell lysates were purchased from Santa Cruz Biotechnology (Santa Cruz, CA, USA). PE-conjugated anti-CD73 mAb (clone TY/23) and its isotype control were purchased from BD Pharmingen (San Jose, CA, USA). Adenosine 5'- $\alpha, \beta$-methylene diphosphate (AOPCP), adenosine receptor agonists [2-chloro- $\mathrm{N}^{6}$-cyclopentyladenosine (CCPA) (towards $\mathrm{A}_{1}$ ), 2-p-(2-carboxyethyl)phenethylamino-5'$\mathrm{N}$-ethylcarboxamidoadenosine hydrochloride hydrate (CGS-21680) (towards $\mathrm{A}_{2 \mathrm{~A}}$ ) and $\mathrm{N}^{6}$-(3-iodobenzyl)adenosine5'-N-methyluronamide (IB-MECA) (towards $A_{3}$ )], cell culture reagents, heparin, hemoglobin and Drabkin's solution were purchased from Sigma-Aldrich (St. Louis, MO, USA). bFGF was purchased from Calbiochem (Darmstadt, Germany) and Matrigel (Basement Membrane Matrix) was purchased from BD Biosciences (Albany, NY, USA).

Mice. Wild-type and CD73-deficient C57BL/6 mice (26) were bred and maintained at the Tri-City Central Animal Laboratory of the Medical University of Gdańsk (Poland) under SPF conditions. Animal studies were performed on male 4- to 8-week-old mice according to EU and national guidelines with the approval of the Local Independent Ethics Committee.

Cell cultures. The B16F10 murine melanoma cell line was kindly provided by Professor J. Konopa (Gdańsk University of Technology, Poland). The HEK293T cell line was purchased from Open Biosystems (Lafayette, CO, USA). Both cell lines were cultured in DMEM supplemented with $10 \%$ FBS in a humidified atmosphere containing $5 \% \mathrm{CO}_{2}$ at $37^{\circ} \mathrm{C}$.

Cell adhesion assay. The wells of a 96-well plate were precoated with $100 \mu \mathrm{l}$ of $16 \mu \mathrm{g} / \mathrm{ml}$ Matrigel, $15 \mu \mathrm{g} / \mathrm{ml}$ fibronectin or $50 \mu \mathrm{g} / \mathrm{ml}$ type I collagen and non-specific binding sites were blocked with $10 \mathrm{mg} / \mathrm{ml}$ denatured BSA. B16F10 cells were grown to $\sim 80 \%$ confluence in DMEM containing $10 \% \mathrm{FBS}$, pre-incubated in serum-free DMEM for $2 \mathrm{~h}$, trypsinized and suspended at a concentration of $3 \times 10^{5}$ cells $/ \mathrm{ml}$. Cell suspension $(100 \mu \mathrm{l})$ in serum-free DMEM was applied to each well for $15 \mathrm{~min}$ (Matrigel) or $30 \mathrm{~min}$ (fibronectin or type I collagen). When indicated, medium was supplemented with $200 \mu \mathrm{M}$ AOPCP and/or $10 \mu \mathrm{M}$ adenosine receptor agonists. Non-adherent cells were subsequently removed by gentle washing with PBS. The relative number of adherent cells was calculated using an MTT metabolic assay. The optical density of wells containing cells incubated only with a serum-free medium was assumed to be $100 \%$ of cell adhesion.

Wound healing assay. B16F10 cells were seeded in a 96-well plate $\left(2 \times 10^{5}\right.$ cells/well) and cultured until $90 \%$ confluent. Cells were subsequently serum-starved overnight, and a linear wound was applied to the monolayer using a $200-\mu 1$ pipette tip. Loose cells were washed away with PBS. When indicated, serum-free DMEM was supplemented with $200 \mu \mathrm{M}$ AOPCP and/or $10 \mu \mathrm{M}$ adenosine receptor agonist. Images were captured immediately after wounding and again after $24 \mathrm{~h}$. The wound width was calculated using arbitrary units with the use of ImageJ software (NIH). The cell migration distance was determined by subtracting the width of the wound after $24 \mathrm{~h}$ from its initial width at time 0 (immediately after wounding). The values were plotted as the percentage of the wound closure, with the initial width set to $0 \%$.

Matrigel invasion assay. The thin coating Matrigel method in a 24-well plate Transwell system was used (27). Transwell membrane inserts (BD Biosciences, Franklin Lakes, NJ, USA) with a pore size of $8 \mu \mathrm{m}$ were pre-coated with $5 \mu \mathrm{g}$ of Matrigel per filter and dried overnight. B16F10 cells were serum-starved

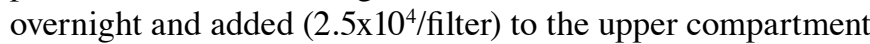
in serum-free DMEM supplemented with $200 \mu \mathrm{M}$ AOPCP and/or $10 \mu \mathrm{M}$ adenosine receptor agonist, when indicated. Conditioned medium from the NIH 3T3 cell culture was added to the lower chamber as a chemoattractant. After $22 \mathrm{~h}$ of incubation, the Matrigel coating on the upper surface of the filter was wiped off using a cotton swab. Cells that migrated through the filter were fixed, stained with Giemsa stain and counted under the microscope (magnification, x400) from eight randomly selected fields for each filter isolated from triplicate chambers.

Western blot analysis. Cells $\left(8 \times 10^{6}\right)$ were lysed in $100 \mu \mathrm{l}$ of lysis buffer (1\% glycerol, 5 mM EDTA, 0.1\% NP40 in PBS and $\mathrm{pH}$ 7.4) containing complete protease inhibitor cocktail (Roche Applied Sciences, Indianapolis, IN, USA) and centrifuged for $10 \mathrm{~min}$ at $17,000 \mathrm{x}$ g. Proteins in the supernatant were denatured by boiling in $5 \mathrm{X}$ Laemmli buffer, subjected to SDS-PAGE electrophoresis and transferred to a PVDF membrane (Immobilon; Millipore, Etten-Leur, The Netherlands). The next steps were performed according to the BM Chemiluminescence Blotting Substrate instructions (Roche Applied Sciences), with an overnight incubation in the primary antibody (mouse anti-CD73 or anti-CD39 mAb) and a 30-min incubation in a secondary goat anti-mouse HRP-conjugated mAb (Santa Cruz Biotechnology). 
Immunofluorescence microscopy. B16F10 cells were plated on glass coverslips and fixed with $4 \%$ paraformaldehyde after $48 \mathrm{~h}$. For detection of internal antigens, cells were permeabilized with $0.1 \%$ Triton $\mathrm{X}-100$ for $3 \mathrm{~min}$. Cells were subsequently blocked for 15 min with $0.5 \%$ BSA in PBS, incubated overnight with a primary mouse anti-CD73 mAb and with the goat anti-mouse secondary Cy3-conjugated antibody (Jackson ImmunoResearch, West Grove, PA, USA) for $30 \mathrm{~min}$. Permeabilized cells were counterstained with $0.05 \%$ DAPI (Sigma) for $5 \mathrm{~min}$. The stained cells were mounted on glass slides in n-propyl-gallate, and images were obtained using an Axiovert Zeiss microscope and AxioVision software (Zeiss, Oberkochen, Germany).

Analysis of cells by flow cytometry. Cells $\left(1 \times 10^{5}\right)$ were collected, suspended in a staining solution $\left(2 \% \mathrm{FBS}, 0.09 \% \mathrm{NaN}_{3}\right.$ in PBS) and incubated for $5 \mathrm{~min}$ at $4^{\circ} \mathrm{C}$. Relevant PE-conjugated antibodies were added to the final concentration of $1 \mu \mathrm{g}$ per $10^{6}$ cells and incubated for $30 \mathrm{~min}$ at $4^{\circ} \mathrm{C}$. Cells were subsequently washed twice with a staining solution and suspended in staining solution for analysis. When the visualization of the intracellular antigen was required, cells were fixed with $4 \%$ paraformaldehyde and treated with permeabilization-wash buffer (BioLegend, San Diego, CA, USA) as an additional step prior to incubation with antibodies. The analysis was performed using an LSR II (Becton-Dickinson, Franklin Lakes, NJ, USA) flow cytometer equipped with an argon ion laser (488 nm). All the measurements were performed for $1 \times 10^{4}$ cells by gating the cells that exhibited the typical forward and side scatter features of non-disintegrated cells. The percentage of positive cells was measured from a cut-off set using an isotype-matched non-specific control antibody. Data were analyzed offline using the BD FACSDiva (BD Biosciences) and Cyflogic (CyFlo Ltd., Turku, Finland).

Tumor inoculation. B16F10 tumor cells were injected subcutaneously into the left flank of syngenic C57BL/6 wild-type or CD73-deficient mice $\left(2.5 \times 10^{6}\right.$ tumor cells/animal). When indicated, the s.c. injection was supplemented with $200 \mu \mathrm{M}$ AOPCP, and AOPCP was subsequently administered via intraperitoneal injection at a dose of $20 \mathrm{mg} / \mathrm{kg}$ on days 5,7 , 9 and 12. Control animals were injected with vehicle only. Tumor volume was monitored with calipers and calculated using the formula: $V=4 / 3 \pi \times(D / 2) \times(d / 2)^{2}$, where $V$ is the volume $\left(\mathrm{mm}^{3}\right), \mathrm{D}$ is the long diameter $(\mathrm{mm})$ and $\mathrm{d}$ is the short diameter ( $\mathrm{mm}$ ). Mice were euthanized after 14 days and tumors were fixed in formalin for subsequent histological analysis.

Matrigel plug angiogenesis assay. Mice were inoculated s.c. on the right side of the linea alba with $600 \mu \mathrm{l}$ of Matrigel (10 mg/ml) mixed with heparin (12.5 U) and bFGF (200 ng), supplemented with $200 \mu \mathrm{M}$ AOPCP or saline (control and $\mathrm{CD}^{-/ /} 3^{-}$plugs). When indicated, the animals were injected every second day around the plug (four injection sites) with AOPCP (100 $\mu \mathrm{l}$ of $200 \mu \mathrm{M}$ solution) or saline. In the cancerinduced angiogenesis assay, the inoculation contained $600 \mu \mathrm{l}$

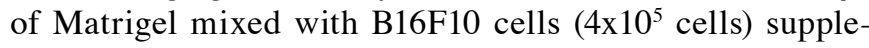
mented with $200 \mu \mathrm{M}$ AOPCP or saline (control plugs). Plugs were removed from euthanized mice after 8 days. Matrigel plug vascularization was quantified by measuring hemoglobin concentration in homogenized plugs using Drabkin's solution according to the manufacturer's instructions.

Histological analysis. Excised tumors were fixed in formalin and embedded in paraffin. Paraffin sections were stained with hematoxylin and eosin using the method of May-GrünwaldGiemsa or were stained with Heidenhain's AZAN modification of Mallory's trichrome stain. Images from microscope slides were acquired with an iPOLiS SNB-7000 digital camera (Samsung, Daegu, China) connected to an Eclipse E800 microscope (Nikon, Tokyo, Japan).

Statistical analysis. Mean values were obtained from at least three separate experiments and reported as the mean ( \pm SD). For statistical analysis, the Mann-Whitney test for two unpaired groups of a non-Gaussian population was used. Advanced hemorrhagic necrosis incidence in tumors was evaluated using Fisher's non-parametric test. P-values $<0.05$ were considered to indicate statistically significant differences.

\section{Results}

B16F10 cells possess significant amounts of CD73 and $C D 39$. To verify the benefits of the chosen cell model for an in vitro analysis of CD73 influence on invasive potential, we evaluated the B16F10 cell line for the presence of both CD39 (NTPDase1), an ectonucleotidase that catalyzes the sequential hydrolysis of ATP to AMP and CD73, which converts AMP to adenosine.

The CD73 content in the whole cell lysate of B16F10 cells (Fig. 1A) was examined by western blot analysis and compared with other tissues and cells exhibiting diverse levels of ecto-5'-nucleotidase enzymatic activity: human melanoma A375 cell line (high level) (12), human breast adenocarcinoma MDA-MB-231 cell line (high level) (17) and human embryonic kidney cell line HEK293T (low level) $(28,29)$. Judging by the intensity and localization of specific bands, the levels of CD73 protein in $\mathrm{B} 16 \mathrm{~F} 10$ cells appeared to be quantitatively and qualitatively comparable to the levels found in other cell lines. The molecular mass of the detected protein was similar to the non-glycosylated form of CD73 (58 kDa) (30). Only lysates prepared from the spleen of mouse strains syngenic (C57BL/6) or non-syngenic (BALB/c) to B16F10 exhibited the additional major band with a higher molecular mass, a characteristic of highly glycosylated CD73 present on lymphocytes (10).

Immunofluorescence staining (Fig. 1C and D) revealed that CD73 was less densely distributed (but still significantly expressed) on the B16F10 cell surface when compared to HEK293T. The use of flow cytometry to detect CD73 with a PE-conjugated anti-CD73 mAb (clone TY/23) did not deliver conclusive results, as cell-surface CD73 was not detectable in non-fixed B16F10 or HEK293T cells, although the intracellular antigen was apparent in the fixed cells (Fig. 2). Targeting either CD73 with AOPCP or various adenosine receptors with specific agonists (IB-MECA, CGS-2180 and CCPA) did not change the total CD73 protein levels or its distribution in B16F10 cells (data not shown).

CD39 content was significantly high and comparable among the whole cell lysates obtained from B16F10 cells or the spleen of a syngenic mouse (Fig. 1B). 

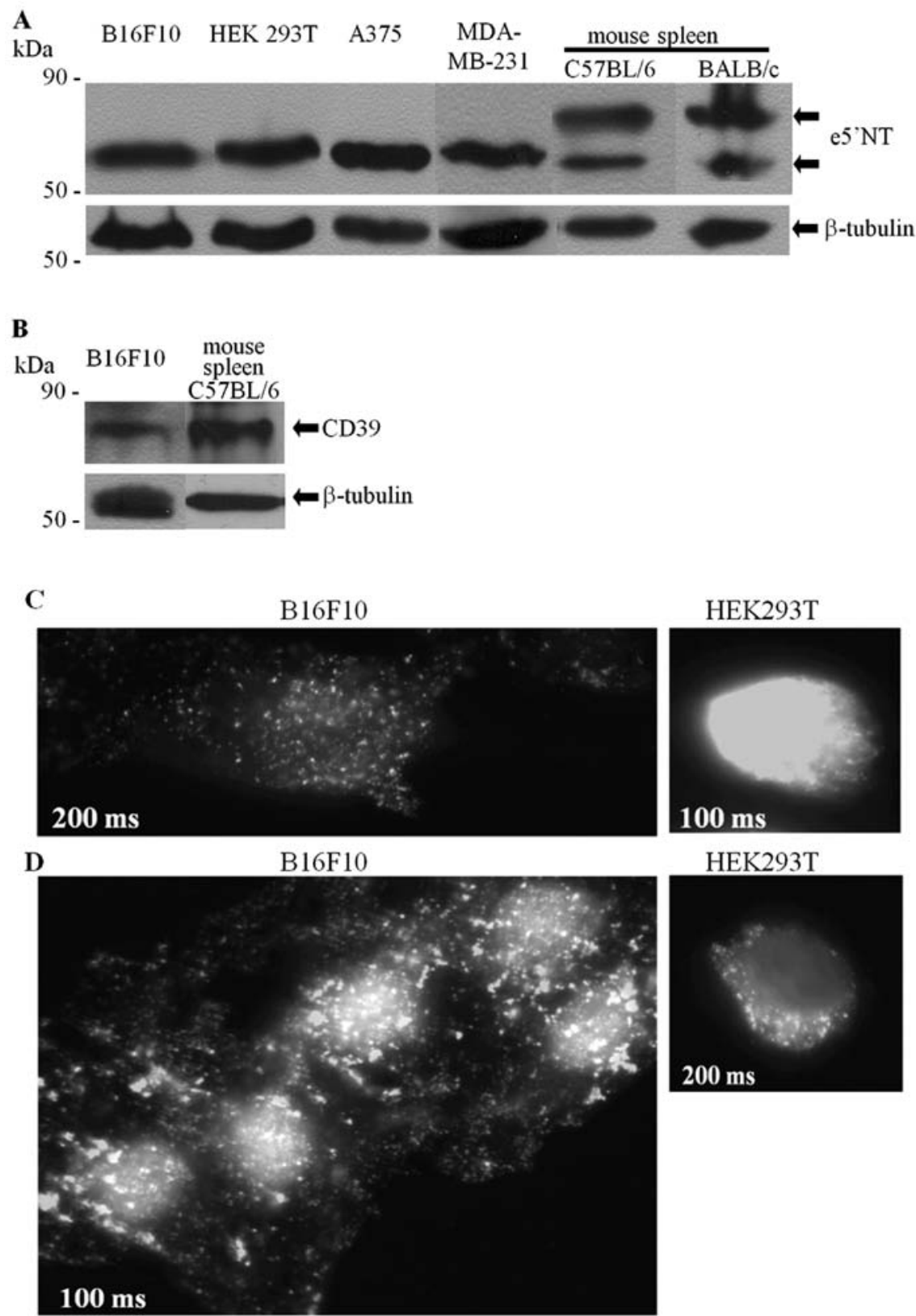

HEK293T

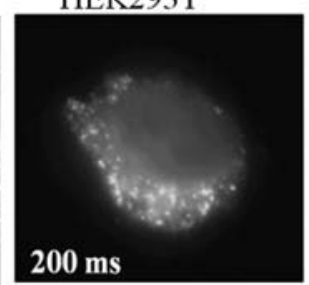

Figure 1. Distribution of CD73 and CD39 in cancer cell lines and normal spleen tissue. (A and B) Whole cell lysates prepared from the indicated cell lines and spleen samples from mice syngenic to the B16F10 cell line (C57Bl/6) and non-syngenic mice (BALB/c) were subjected to western blot analysis with mAbs specific to murine/human CD73 (A) or murine CD39 (B). The positions of proteins and molecular size standards are indicated. $\beta$-tubulin served as a loading control. (C and D) Immunofluorescence microscopy for the surface (C) and intracellular (counterstained with DAPI) (D) expression of CD73 in B16F10 and HEK293T cells (x400). For some images, the time of exposure was shortened to $100 \mathrm{~ms}$ as high fluorescence intensity at $200 \mathrm{~ms}$ did not allow for the clear visualization of details. The data in all figures are representative of three to five independent experiments.

Targeting CD73 with AOPCP modulates B16F10 melanoma cell ability to adhere to the ECM, migrate and invade in vitro. We analyzed the ability of melanoma cells to adhere to ECM proteins, migrate and invade. The specific competitive CD73 inhibitor AOPCP, a hydrolytically stable analog of ADP, was used for this purpose. Selective adenosine receptor agonists $\operatorname{CCPA}\left(\mathrm{A}_{1} \mathrm{R}\right)$, CGS-21680 $\left(\mathrm{A}_{2 \mathrm{~A}} \mathrm{R}\right)$ and IB-MECA $\left(\mathrm{A}_{3} \mathrm{R}\right)$ were used in the presence of AOPCP to determine which of the adenosine receptors was involved in mediating these processes. Although all the agonists, AOPCP and serum-starvation were tested, none of these treatments influenced the viability of B16F10 cells under the experimental conditions described (data not shown).
B16F10 cell adherence to Matrigel and fibronectin was measured (Fig. 3A). The major component of the Matrigel basement membrane matrix is laminin, but Matrigel also contains type IV collagen, heparan sulfate proteoglycans and entactin/nidogen. Attachment of B16F10 cells to the matrix was significantly inhibited by AOPCP and was $\sim 51 \%$ of the control $(\mathrm{P}<0.05)$. Melanoma attachment to fibronectin displayed a larger decrease of $\sim 32 \%$ of the control ( $\mathrm{P}<0.005)$. Blocking CD73 by AOPCP was $\sim 20 \%( \pm 2 \%)$ stronger $(\mathrm{P}<0.01)$ when compared to the blocking ability of monoclonal anti-CD73 antibodies (data not shown). No significant changes in the ability of the melanoma cells to attach to ECM proteins were observed upon the simultaneous addition of adenosine receptor agonists and AOPCP. 

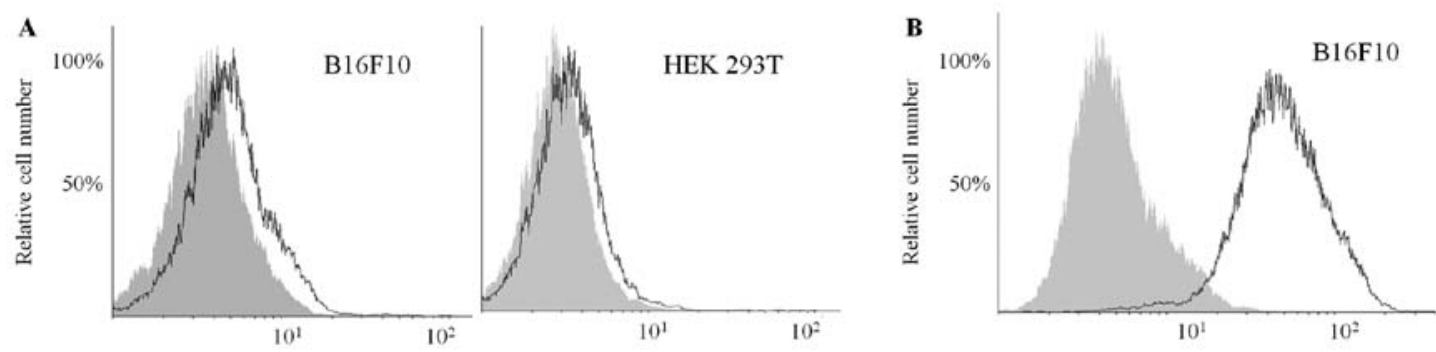

Figure 2. Flow cytometric analysis of CD73 in B16F10 and HEK293T cell lines. Cells were stained with a PE-conjugated anti-CD73 mAb to analyze surface (A) and intracellular (B) expression of CD73. Gray field, control unstained; black line, CD73. The data in all figures are representative of three independent experiments.
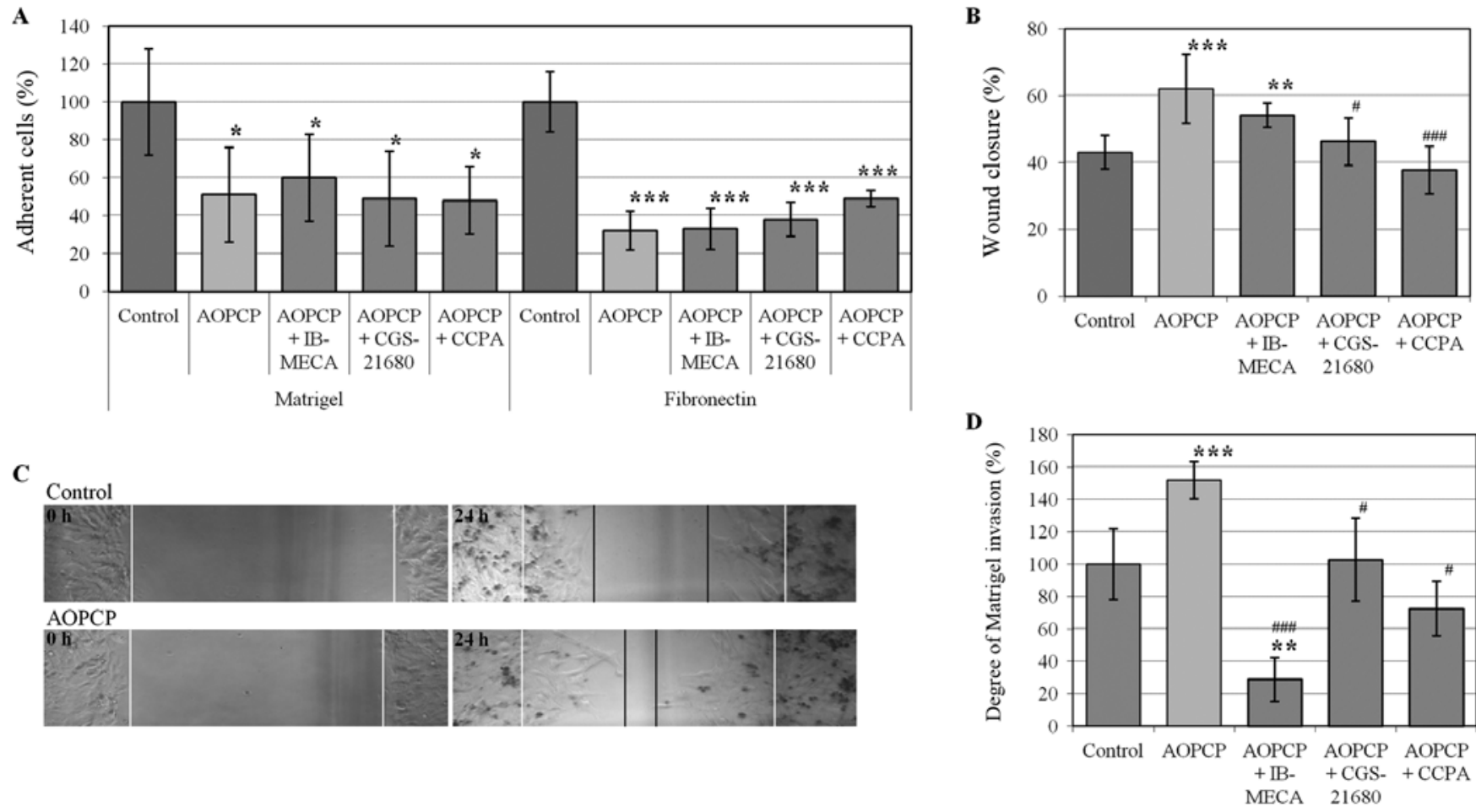

Figure 3. Changes in B16F10 cell adhesion to various ECM proteins, migration and invasion in response to CD73 inhibitor and various adenosine receptor agonists. The ability of B16F10 cells to adhere to Matrigel or fibronectin (A), to migrate in a wound healing assay (B and C) and to invade Matrigel in Boyden chambers (D) was examined by incubating with $200 \mu \mathrm{M}$ AOPCP or $10 \mu \mathrm{M}$ adenosine receptor agonists. Graph A shows the inhibitory effect of AOPCP on the attachment of B16F10 cells to Matrigel or fibronectin as independent of adenosine receptor stimulation. Graph B shows the cell motility-inducing effects of AOPCP and its reversal by the addition of adenosine receptor agonists as a percentage of the wound closure after $24 \mathrm{~h}$. Graph $\mathrm{C}$ is a representative image for non-treated and AOPCP-treated cells with the wound borders at a starting point (marked with the white lines) and after $24 \mathrm{~h}$ (marked with the black lines). Graph D shows the increase in the invasive potential of AOPCP-treated B16F10 cells and its decrease by the addition of various adenosine receptor agonists compared to the non-treated control ( 70 cells/field). All data points represent averages of three to four independent tests. Standard deviations are marked. ${ }^{*} \mathrm{P}<0.05,{ }^{* *} \mathrm{P}<0.01,{ }^{* * *} \mathrm{P}<0.005$ vs. untreated control. ${ }^{\#} \mathrm{P}<0.05,{ }^{\# \# \#} \mathrm{P}<0.005$ vs. AOPCP treated cells.

The wound healing assay was used to investigate directional cell migration after breaking the continuity of a monolayer. Addition of AOPCP to the medium significantly increased the motility of B16F10 cells, as demonstrated by an increase in the wound closure from $43 \%$ in control cells to $62 \%$ upon AOPCP treatment $(\mathrm{P}<0.005) 24 \mathrm{~h}$ after wound formation (Fig. 3B and $\mathrm{C}$ ). Addition of adenosine $\mathrm{A}_{1}$ or $\mathrm{A}_{2 \mathrm{~A}}$ receptor agonists reversed the effects of AOPCP $(\mathrm{P}<0.005$ or $\mathrm{P}<0.05$, respectively). Upon stimulation of the $\mathrm{A}_{3}$ receptor, the observed effects were not statistically significant $(\mathrm{P}=0.11)$ (Fig. 3B).

The rate of Matrigel invasion was quantified via the use of a Transwell chamber (Boyden) (Fig. 3D). The number of cells migrating through a membrane coated with Matrigel towards a chemotactic agent was increased by $\sim 50 \%$ in the presence of AOPCP $(\mathrm{P}<0.005)$. This effect was completely reversed by simultaneous stimulation of adenosine receptors of type $A_{1}$ or $\mathrm{A}_{2 \mathrm{~A}}(\mathrm{P}<0.05)$ and significantly diminished below the control level by stimulation of $\mathrm{A}_{3} \mathrm{R}(\mathrm{P}<0.01$ compared to the control).

B16F10 melanoma growth in vivo is inhibited in CD73deficient mice. We compared the melanoma growth rates in the wild-type and CD73-deficient mice to discriminate between the influence of the host cell- and cancer cell-derived CD73 on tumor growth. B16F10 tumor cells were injected subcutaneously and the tumor growth was monitored during the early phase of its growth (up to 14 days after inoculation). As shown 


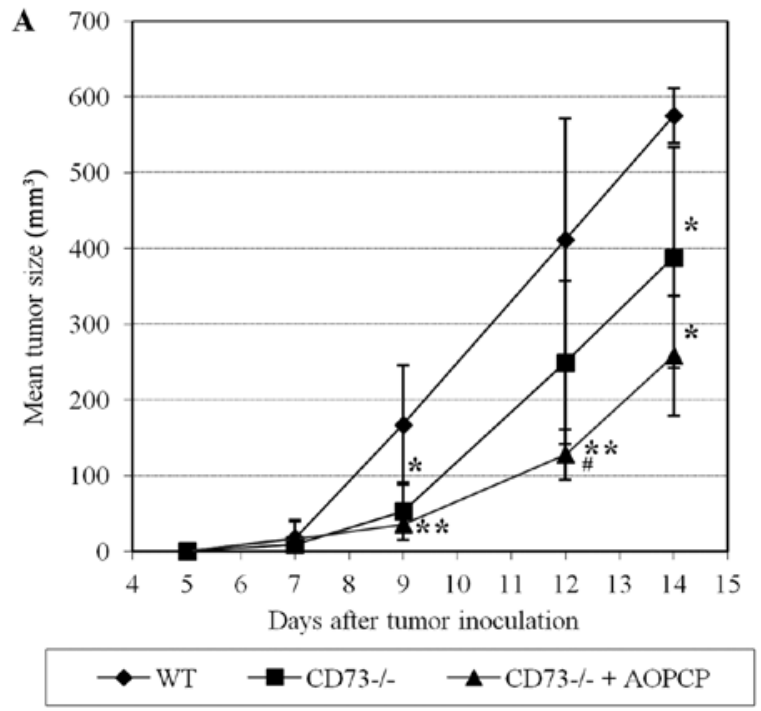

B
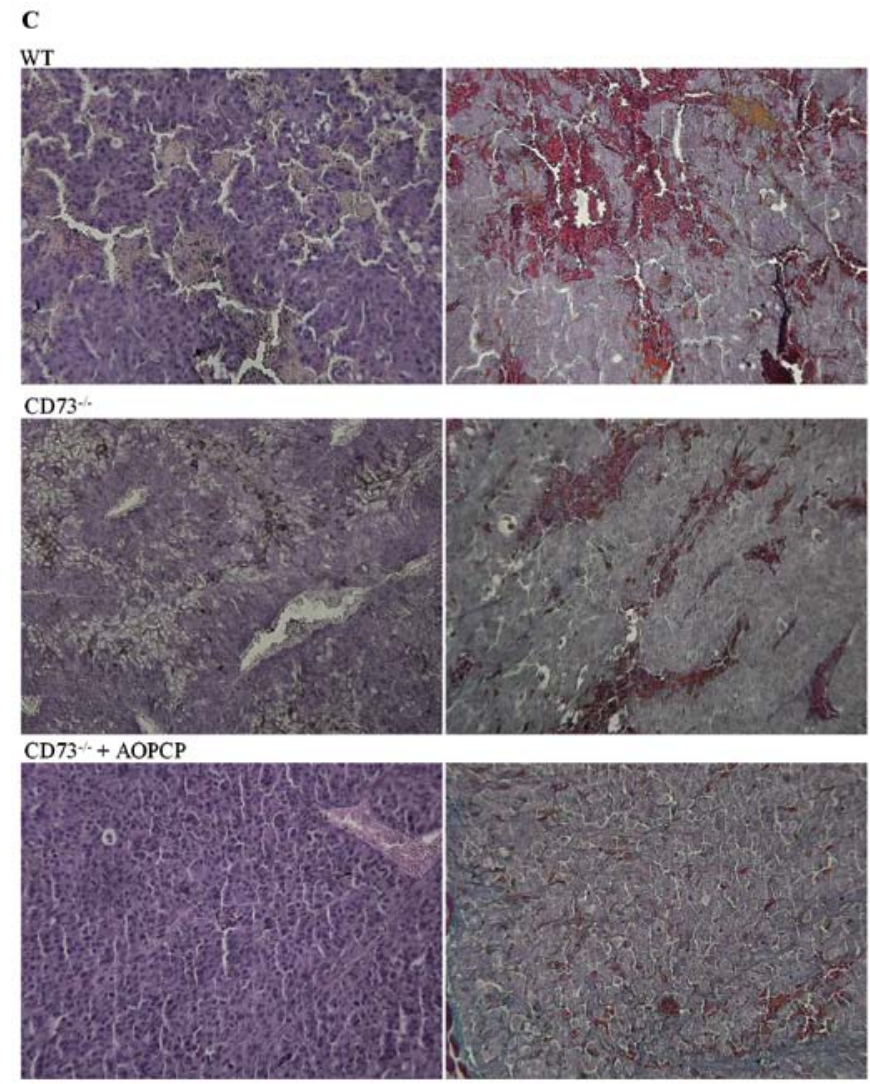

Figure 4. Growth rate and histological analysis of B16F10 melanoma in the wild-type and CD73 ${ }^{-/}$mice in the absence or presence of AOPCP. Wild-type and CD73-homozygous deficient mice were injected subcutaneously with $2.5 \times 10^{6} \mathrm{~B} 16 \mathrm{~F} 10$ cells and monitored for 14 days after injection. In the third group, injected B16F10 cells were supplemented with $200 \mu \mathrm{M}$ AOPCP and then AOPCP at a dose $20 \mathrm{mg} / \mathrm{kg}$ was applied i.p. on days: 5, 7, 9 and 12. Data represent means of five or six mice per group. (A) Changes in the tumor volume growth rate during experiments. Standard deviations are marked. $\mathrm{P}<0.05$, ${ }^{* *} \mathrm{P}<0.01$ vs. $\mathrm{WT}$ ${ }^{\#} \mathrm{P}<0.05$ vs. CD73 ${ }^{-1}$. (B) Incidence of hemorrhagic necrosis in tumors on day 14. (C) Images of representative areas from tumor paraffin slides stained with hematoxylin and eosin (left panel) and Azan-Mallory (right panel) (x200).

in Fig. 4A, the tumor growth rate was significantly reduced in CD73-deficient mice ( $P<0.05$, days 9 and 14 after inoculation). Simultaneous targeting of CD73 on tumor cells with AOPCP further reduced the tumor growth rate when compared to both the wild-type mice $(\mathrm{P}<0.01$, day $9 ; \mathrm{P}<0.05$, days 12 and 14) and the CD73-deficient mice not treated with AOPCP $(\mathrm{P}<0.05$, day 12). No lung metastases were observed within 14 days of the subcutaneous injection.

The development of local blood vessels is commonly recognized as a growth-limiting mechanism for neoplasms and the process of angiogenic switch has previously been found to be adenosine-dependent (31). In view of these reports, we assessed whether the decrease in the tumor growth rate in CD73-deficient mice with CD73-inhibited cancer cells was directly related to a decrease in angiogenic response. This hypothesis was supported by our observation of advanced hemorrhagic necrosis (manifested as a tumor perforation and a blood clot on its surface). Incidence of such necrosis was significantly higher $(\mathrm{P}<0.05) 14$ days after tumor inoculation in CD73-deficient hosts (38\% of untreated and $50 \%$ of AOPCP-treated mice) when compared to the wild-type animals (no animals exhibited necrosis on day 14) (Fig. 4B). This hypothesis was also supported by histological analysis of tumors (Fig. 4C) by H\&E or Azan-Mallory staining. We observed a visible decrease in the vascular density of tumors isolated from AOPCP-treated CD73-deficient mice when compared to the highly vascularized tumors observed in both wild-type controls and CD73-deficient mice not treated with AOPCP (no significant difference was observed between the two latter groups).

The angiogenic response of the host and B16F10 melanomainduced angiogenesis are reduced upon CD73 inhibition. To analyze a putative role of CD73 in in vivo angiogenesis, the Matrigel plug angiogenesis assay was used. The assay was performed by adding CD73 inhibitor (AOPCP) locally to the plug in a wild-type animal or by using CD73-deficient hosts. Neovascularization of the plug was analyzed 8 days after injection when the blood vessel formation was robust enough for hemoglobin measurements and the total volume of the angiogenic blood vessels in a Matrigel plug could be quantified.

Substantial ingrowth of new blood vessels into the Matrigel plug was observed in wild-type mice in the presence of proangiogenic bFGF. A single application of AOPCP decreased the volume of blood in new vessels; however, this result was not 
A

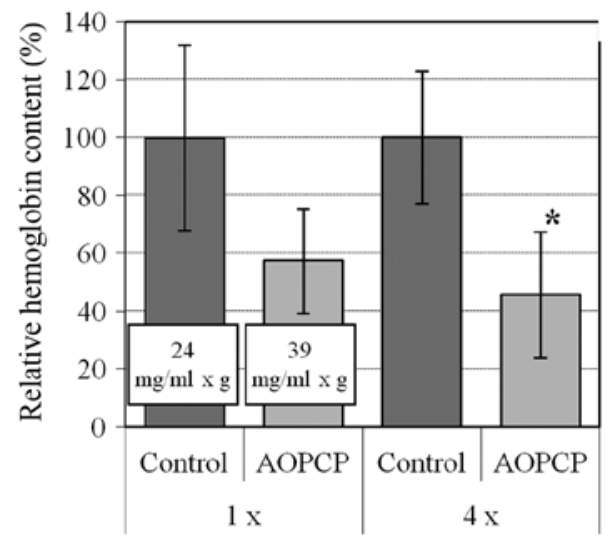

B

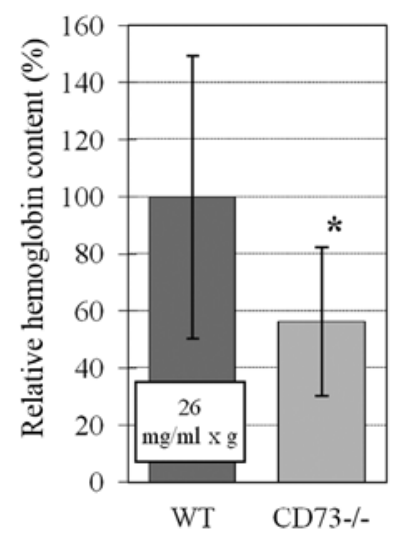

C

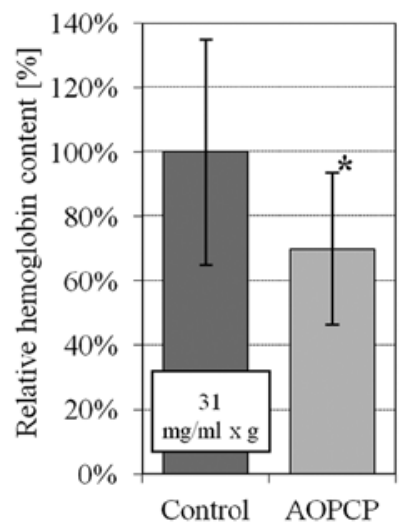

Figure 5. The relative changes of hemoglobin content in subcutaneous Matrigel plugs in the absence or presence of $\mathrm{AOPCP}$ or in $\mathrm{CD} 73^{-/-}$mice. Graph $\mathrm{A}$ shows an inhibitory effect of AOPCP supplementation on the hemoglobin content of Matrigel plugs in WT mice. AOPCP or saline (for control) for the single application $(1 \mathrm{x})$ were added as a supplement to Matrigel plugs before injection (data represents means for six mice per group). In the second group (4x), four consecutive injections of AOPCP (or saline for control) were injected every second day around the Matrigel plug (seven mice per group). ${ }^{*}<0.05$. Graph $\mathrm{B}$ compares hemoglobin content of Matrigel plugs in WT and CD73-deficient mice. Vascularization of the plugs in CD73-homozygously deficient mice was significantly decreased (eight mice per group). ${ }^{*} \mathrm{P}<0.05$. Graph $\mathrm{C}$ shows an inhibitory effect of AOPCP supplementation (or saline for control) on a hemoglobin content of Matrigel plugs injected together with $4 \times 10^{5}$ B16F10 melanoma cells into WT mice. There were no additional injections of AOPCP. * $<0.05$. Data represent the changes in the mean total volume of blood vessels overgrowing the plugs. The vascularization of plugs (presented in the insets) was measured as hemoglobin concentration $(\mathrm{mg} / \mathrm{ml})$ calculated per gram of plug weight $(\mathrm{g})$. Standard deviations are indicated.

statistically significant $(\mathrm{P}=0.09)$. Statistical significance was achieved after four consecutive local injections of AOPCP around the plug, which decreased the total volume of blood vessels to $\sim 46 \%$ of the control $(\mathrm{P}<0.05)$ (Fig. 5A). Moreover, we observed that neoangiogenesis was compromised in CD73deficient mice when compared to wild-type mice, with the total blood volume in plugs exhibiting a significant decrease to $\sim 56 \%$ of wild-type mice $(\mathrm{P}<0.05)$ (Fig. $5 \mathrm{~B})$.

When B16F10 melanoma cells were introduced into a Matrigel plug in wild-type animals, the angiogenic response was strong ( $\sim 31 \mathrm{mg} / \mathrm{ml} \mathrm{x} \mathrm{g})$ and the ingrowth of tumor blood vessels was significantly decreased to $\sim 60 \%$ of the control values after a single application of AOPCP (Fig. 5C).

Thus, we found that formation of new vessels in C57BL/6 mice and tumor-induced angiogenesis in Matrigel plugs is inhibited by decreasing CD73 enzymatic activity.

\section{Discussion}

Recently published reports confirmed the association between CD73 and tumor progression in melanoma $(9,12,15,16)$. The authors claim that either CD73-deficiency in transgenic mice or a pharmacological inhibition of CD73 in wild-type mice can protect against the development of subcutaneous tumors and experimental lung metastases $(9,15,16)$. However, murine B16F10 melanoma was characterized by some authors as a CD73-negative cell line despite earlier reports that low, but significant, cell surface 5'-nucleotidase activity was present $(24,25)$. In the present study, we analyzed CD73 expression in B16F10 cells using various techniques: western blotting, immunofluorescence microscopy and flow cytometry with two different antibodies (non-conjugated and $\mathrm{PE}$-conjugated $\mathrm{mAb}$ ). These results convincingly showed that B16F10 cells contain ecto-5'-nucleotidase levels comparable to those found in high CD73-expressing human melanoma
A375 (12) or breast adenocarcinoma MDA-MB-231 cell lines (17). The protein displayed a similar molecular mass in all analyzed cell lines, which was smaller than the more heavily glycosylated form present on T lymphocytes (10). A significant amount of CD73 was detected on the B16F10 cell surface by immunofluorescence; however, its main localization pattern appeared to be intracellular. This is in agreement with an earlier hypothesis stating that an extensive intracellular distribution of CD73 is present in a membrane-bound pool (i.e., lysosomes, Golgi apparatus and transcytotic vesicles) and that CD73 undergoes continual exchange between the plasmatic and internal membranes $(1,32,33)$. We encountered a problem detecting CD73 on the surface of non-fixed B16F10 and HEK293T cells by flow cytometry (although the intracellular antigen was apparent in the fixed cells), but this might be due to ecto-domain shedding after binding of the anti-CD73 antibody. Such an effect was demonstrated by Airas et al (34) when CD73 expression was examined on the surface of lymphocytes.

The paradigm for the role of CD73 in tumor progression has not yet been established. In the literature, examples suggesting that a high level of CD73 expression on tumor cells can be used as either a negative (19) or a positive (23) prognostic marker can be found. However, an explicitly positive correlation between CD73 expression and invasive potential was shown in some cancer cells in vitro. Overexpression of CD73 in human breast cancer cells increased their ability to invade, migrate and adhere to the ECM, thus upregulating their metastatic potential $(17,20)$. For the highly invasive human melanoma cell lines, it was shown that CD73 was upregulated and correlated with a number of metastasis-related markers (12). On the contrary, some other clinical research studies showed reduced CD73 activity in breast cancer cells when compared to the adjacent non-involved tissue $(21,22)$. Additionally, the reverse correlation between CD73 activity 
and metastatic potential was occasionally indicated for rat breast cancer cell lines (35) and B16F10 cells $(24,25)$. In the present study, we showed that the highly invasive murine B16F10 cells contained levels of CD73 protein comparable to those found in highly invasive human melanoma A375 (12) and human breast adenocarcinoma MDA-MB-231 cell lines (17). Moreover, the targeting of CD73 in B16F10 cells with a selective inhibitor led to a significant increase in their invasive potential in vitro.

It should be noted that blocking CD73 with AOPCP decreased B16F10 cell adherence to fibronectin and to the basement membrane matrix (Matrigel) and that this adherence was not modulated via adenosine receptor signaling. This is particularly significant with regards to the decisive role of cell adherence to ECM components in the escape and invasion of ectopic environments (36). It has been shown that isolated CD73 binds to fibronectin in a specific and saturable manner $(11,37)$. Such specificity suggests a direct role of CD73 in the binding of B16F10 cells to fibronectin. It is possible that the conformation of ecto-5'-nucleotidase changed in the presence of inhibitory AOPCP, thus interfering with ECM binding, similar to previously indicated results for eukaryotic cells and the 5'-nucleotidase from E. coli (38). The difference between its effect on B16F10 adhesion to Matrigel and fibronectin might be explained by the presence of laminin in Matrigel and its ability to direct more CD73 to the cell surface (39). The adherence-dependent processes of migration and invasion were upregulated in AOPCP-treated B16F10 cells and downregulated by a set of adenosine receptor agonists. Therefore, the involvement of CD73 in melanoma cell invasion was confirmed and probably occurs via direct CD73-mediated interactions with ECM constituents. Nevertheless, the exact molecular mechanisms of this phenomenon require further study.

By contrast, in vivo experiments showed that CD73deficiency in the host caused impaired tumor growth in mice. Additionally, pharmacological inhibition of CD73 activity on the grafted B16F10 melanoma in CD73-deficient hosts displayed even further decreases in tumor growth. Earlier reports suggest that $\mathrm{B} 16 \mathrm{~F} 10$ melanoma growth is slower in CD73-deficient mice when compared to a wild-type mouse and that this effect was attributable to the immunomodulatory role of the host-derived CD73 on lymphocytes $(9,15,16)$. We propose here a similar role for the angiogenic switch, a critical step in melanoma tumor growth correlating with the transition to a metastatic phase (31); it also correlates with an increase in tumor thickness and resultant hypoxia (40). Adenosine is regarded as an important hypoxia-counteracting mediator $(41,42)$ which exerts a mitogenic effect on the endothelium and regulates the synthesis of pro- and anti-angiogenic factors (such as VEGF through the $\mathrm{A}_{3}$ receptor in melanoma cells) $(6,43)$. Experiments performed on human breast cancer xenografts in a nude mouse model have demonstrated that both the chemical inhibition of CD73 and systemic CD73deficiency can decrease angiogenesis not only in vitro (in isolated pulmonary microvascular endothelial cells) but also in vivo by contributing to an increase in the necrotic area of the tumor $(17,18)$. The regulatory role of adenosine produced extracellularly by the grafted B16F10 cells could be variable. Therefore, we suggested that full inhibition of the adenosine producing enzyme may lead to decreased neoangiogenesis. The histological analysis of the B16F10 tumors have shown that the complete lack of active CD73 in host and grafted cells induced quite significant changes in the vascular density, similar to the results obtained from breast cancer xenografts $(17,18)$, and also led to an increase in necrosis of the tumor tissue (18). However, even the low level expression of CD73 in B16F10 cells in CD73-deficient mice was enough to abrogate this effect, similar to the results described by Yegutkin et al (15). Nevertheless, the increase in the visible hemorrhagic necrosis in this experimental group suggests some less visible changes in tumor vasculature as the lower vascular density correlates with the lower perfusion rate of the tumor tissue, which generally increases the incidence of necrosis (44). These changes remain under investigation.

The Matrigel plug angiogenesis assay is one of the most frequently used angiogenesis assays in current practice (45), and further analysis demonstrated a significant decrease in the total volume of new blood vessels due to both CD73 inhibition in wild-type mice (also in the presence of B16F10 cells) or as a result of CD73-deficiency in animals. Therefore, we propose that inhibition of CD73 in vivo does interfere with the development of the melanoma vascular bed, possibly by influencing either the number or the maturation status of the developing blood vessels. The mechanism has yet to be fully clarified, but aside from a direct influence on vessels, these effects may be mediated through the modulation of some known B16F10 function (e.g., VEGF production), such as those shown by Pötgens et al for the human melanoma xenografts (46).

In conclusion, the present study strongly supports a longstanding hypothesis that CD73 is one of the most important molecules regulating the progression of melanoma. However, a more complex set of interactions was identified in the present study. These results suggest that the membrane enzyme itself and its product, adenosine, can influence the progression by various mechanisms, some of which are contradictory. The ecto-enzyme can function as an adhesion protein and by itself influence melanoma progression. The adenosine product alone can signal to inhibit migration and invasion of melanoma cells, while simultaneously contributing to the development of tumor vasculature. Therefore, final outcomes regarding CD73 influence on tumor progression may be the result of maintaining a critical balance between its enzymatic and nonenzymatic activities regulating many aspects of the invasive potential of tumor cells. Further in-depth studies of CD73 and adenosine receptor signaling will provide us with solid premises regarding the functions of CD73 and which stages of tumor development should be targeted in cancer therapy.

\section{Acknowledgements}

The present study was supported by the W-732 intramural grant from the Medical University of Gdansk (2007-2008), the core fund of the Intercollegiate Faculty of Biotechnology (2008-2010) and the grant N N401 006938 from the National Science Centre (2010-2013). This research was co-funded by the European Social Fund POKL.04.01.01-00-017/10-00 (to M.G.) 'We educate the best - a comprehensive program of development of graduate students, young doctors and academic teaching staff of the University of Gdańsk'. 


\section{References}

1. Zimmermann B: 5'-Nucleotidase: molecular structure and functional aspects. Biochem J 285: 345-365, 1992.

2. Bianchi V and Spychala J: Mammalian 5'-nucleotidases. J Biol Chem 278: 46195-46198, 2003

3. Bly J, White TD and Hoskin DW: The extracellular fluid of solid carcinomas contains immunosuppressive concentrations of adenosine. Cancer Res 57: 2602-2605, 1997.

4. Merighi S, Baraldi PG, Gessi S, Iannotta V, Klotz KN, Leung E, Mirandola P, Tabrizi MA, Varani K and Borea PA: Adenosine receptors and human melanoma. Drug Dev Res 58: 377-385, 2003

5. Bergers G and Benjamin LE: Tumorigenesis and the angiogenic switch. Nat Rev Cancer 3: 401-410, 2003.

6. Auchampach JA: Adenosine receptors and angiogenesis. Circ Res 101: 1075-1077, 2007.

7. Fishman P, Bar-Yehuda S, Synowitz M, Powell JD, Klotz KN, Gessi S and Borea PA: Adenosine receptors and cancer. In: Adenosine Receptors in Health and Disease, Handbook of Experimental Pharmacology. Wilson CN and Mustafa SJ (eds) Springer-Verlag, Berlin, pp399-441, 2009.

8. Stagg J and Smyth MJ: Extracellular adenosine triphosphate and adenosine in cancer. Oncogene 29: 5346-5358, 2010.

9. Stagg J, Divisekera U, Duret H, Sparwasser T, Teng MW Darcy PK and Smyth MJ: CD73-deficient mice have increased anti-tumor immunity and are resistant to experimental metastasis. Cancer Res 71: 2892-2900, 2011

10. Resta R, Yamashita Y and Thompson LF: Ecto-enzyme and signaling functions of lymphocyte CD73. Immunol Rev 161: 95-109, 1998

11. Stochaj U, Dieckhoff J, Mollenhauer J, Cramer M and Mannherz HG: Evidence for the direct interaction of chicken gizzard 5'-nucleotidase with laminin and fibronectin. Biochim Biophys Acta 992: 385-392, 1989.

12. Sadej R, Spychala J and Skladanowski AC: Expression of ecto5'-nucleotidase (eN, CD73) in cell lines from various stages of human melanoma. Melanoma Res 16: 213-222, 2006.

13. Orend G and Chiquet-Ehrismann R: Adhesion modulation by antiadhesive molecules of the extracellular matrix. Exp Cell Res 261: 104-110, 2000.

14. Sadej R, Inai K, Rajfur Z, Ostapkowicz A, Kohler J, Skladanowski AC, Mitchell BS and Spychala J: Tenascin C interacts with Ecto-5'-nucleotidase (eN) and regulates adenosine generation in cancer cells. Biochim Biophys Acta 1782: 35-40, 2008

15. Yegutkin GG, Marttila-Ichihara F, Karikoski M, Niemelä J, Laurila JP, Elima K, Jalkanen S and Salmi M: Altered purinergic signaling in CD73-deficient mice inhibits tumor progression. Eur J Immunol 41: 1231-1241, 2011.

16. Forte G, Sorrentino R, Montinaro A, Luciano A, Adcock IM Maiolino P, Arra C, Cicala C, Pinto A and Morello S: Inhibition of CD73 improves B cell-mediated anti-tumor immunity in a mouse model of melanoma. J Immunol 189: 2226-2233, 2012.

17. Zhou X, Zhi X, Zhou P, Chen S, Zhao F, Shao Z, Ou Z and Yin L: Effects of ecto-5'-nucleotidase on human breast cance cell growth in vitro and in vivo. Oncol Rep 17: 1341-1346, 2007.

18. Wang L, Tang S, Wang Y, Xu S, Yu J, Zhi X, Ou Z, Yang J, Zhou P and Shao Z: Ecto-5'-nucleotidase (CD73) promotes tumor angiogenesis. Clin Exp Metastasis 30: 671-680, 2013.

19. Eroglu A, Canbolat O, Demirci S, Kocaoglu H, Eryavuz Y and Akgül H: Activities of adenosine deaminase and 5'-nucleotidase in cancerous and noncancerous human colorectal tissues. Med Oncol 17: 319-324, 2000

20. Wang L, Zhou X, Zhou T, Ma D, Chen S, Zhi X, Yin L, Shao Z, Ou Z and Zhou P: Ecto-5'-nucleotidase promotes invasion, migration and adhesion of human breast cancer cells. J Cancer Res Clin Oncol 134: 365-372, 2008

21. Pohl AL, Reiner G, Kolb R, Sauermann G, Moser KV and Spona J: Enzyme activities in human breast tumor cells and sera. Cancer Detect Prev 8: 57-66, 1985.

22. Krüger KH, Thompson LF, Kaufmann $\mathrm{M}$ and Möller P Expression of ecto-5'-nucleotidase (CD73) in normal mammary gland and in breast carcinoma. Br J Cancer 63: 114-118, 1991

23. Supernat A, Markiewicz A, Welnicka-Jaskiewicz M, Seroczynska B, Skokowski J, Sejda A, Szade J, Czapiewski P, Biernat $\mathrm{W}$ and Zaczek A: CD73 expression as a potential marker of good prognosis in breast carcinoma. Appl Immunohistochem Mol Morphol 20: 103-107, 2012.
24. Raz A, McLella WL, Hart IR, Bucana CD, Hoyer LC, Sela BA, Dragsten P and Fidler IJ: Cell surface properties of B16 melanoma variants with differing metastatic potential. Cancer Res 40: 1645-1651, 1980.

25. Schroeder F and Gardiner JM: Membrane lipids and enzymes of cultured high-and low-metastatic B16 melanoma variants. Cancer Res 44: 3262-3269, 1984

26. Koszalka P, Özüyaman B, Huo Y, Zernecke A, Flögel U, Braun N, Buchheiser A, Decking UKM, Smith ML, Sévigny J, Gear A, Weber A, Molojavyi A, Ding Z, Weber C, Ley K, Zimmermann H, Gödecke A and Schrader J: Targeted disruption of cd73/ecto5 '-nucleotidase alters thromboregulation and augments vascular inflammatory response. Circ Res 95: 814-821, 2004.

27. Shaw LM: Tumor cell invasion assays: methods in molecular biology. In: Cell Migration: Developmental Methods and Protocols. Guan JL (ed). Vol 294. Humana Press Inc., New York, pp97-105, 2005.

28. Morabito L, Montesinos MC, Schreibman DM, Balter L, Thompson LF, Resta R, Carlin G, Huie MA and Cronstein BN: Methotrexate and sulfasalazine promote adenosine release by a mechanism that requires ecto-5'-nucleotidase-mediated conversion of adenine nucleotides. J Clin Invest 101: 295-300, 1998.

29. St Hilaire C, Ziegler SG, Markello TC, et al: NT5E mutations and arterial calcifications. N Engl J Med 364: 432-442, 2011

30. Flocke $\mathrm{K}$ and Mannherz HG: Isolation and characterization of 5'-nucleotidase of a human pancreatic tumor cell line. Biochim Biophys Acta 1076: 273-281, 1991

31. Ria R, Reale A, Castrovilli A, Mangialardi G, Dammacco F Ribatti D and Vacca A: Angiogenesis and progression in human melanoma. Dermatol Res Pract, Article ID 185687, doi: 10.1155/ 2010/185687, 2010

32. Widnell CC, Schneider YJ, Pierre B, Baudhuin P and Trout A: Evidence for a continual exchange of 5'-nucleotidase between the cell surface and cytoplasmic membranes in cultured rat fibroblasts. Cell 28: 61-70, 1982

33. van den Bosch R, Geuze HJ, du Maine APM and Strous GJ Transport and metabolism of 5'-nucleotidase in a rat hepatoma cell line. Eur J Biochem 160: 49-54, 1986

34. Airas L, Niemelä J, Salmi M, Puurunen T, Smith DJ and Jalkanen S: Differential regulation and function of CD73, a glycosyl-phosphatidylinositol-linked 70-kD adhesion molecule, on lymphocytes and endothelial cells. J Cell Biol 136: 421-431, 1997.

35. Chatterjee SK, Kim U and Bielat K: Plasma membrane associated enzymes of mammary tumors as the biochemical indicators of metastasizing capacity. Analyses of enriched plasma membrane preparations. Br J Cancer 33: 15-26, 1976.

36. Lugassy C, Vernon SE, Busam K, Engbring JA, Welch DR, Poulos EG, Kleinman HK and Barnhill RL: Angiotropism of human melanoma: studies involving in transit and other cutaneous metastases and the chicken chorioallantoic membrane: implications for extravascular melanoma invasion and metastasis. Am J Dermatopathol 28: 187-193, 2006.

37. Stochaj U, Richter H and Mannherz HG: Chicken gizzard 5'-nucleotidase is a receptor for the extracellular matrix component fibronectin. Eur J Cell Biol 51: 335-338, 1990.

38. Sträter N: Ecto-5'-nucleotidase: Structure function relationships Purinergic Signal 2: 343-350, 2006.

39. Méhul B, Aubery M, Mannherz HG and Codogno P: Dual mechanism of laminin modulation of ecto-5'-nucleotidase activity. J Cell Biochem 52: 266-274, 1993.

40. Murphy BJ: Regulation of malignant progression by the hypoxiasensitive transcription factors HIF-1a and MTF-1. Comp Biochem Physiol B Biochem Mol Biol 139: 495-507, 2004.

41. Ohta A and Sitkovsky M: Role of G-protein-coupled adenosine receptors in downregulation of inflammation and protection from tissue damage. Nature 414: 916-920, 2001.

42. Spychala J: Tumor-promoting functions of adenosine. Pharmacol Ther 87: 161-173, 2000

43. Merighi S, Simioni C, Gessi S, Varani K, Mirandola P, Tabrizi MA, Baraldi PG and Borea PA: $\mathrm{A}_{2 \mathrm{~B}}$ and $\mathrm{A}_{3}$ adenosine receptors modulate vascular endothelial growth factor and interleukin- 8 expression in human melanoma cells treated with etoposide and doxorubicin. Neoplasia 11: 1064-1073, 2009.

44. Tufto I, Lyng H and Rofstad EK: Vascular density in human melanoma xenografts: relationship to angiogenesis, perfusion and necrosis. Cancer Lett 123: 159-165, 1998 .

45. Norrby K: In vivo models of angiogenesis. J Cell Mol Med 10: 588-612, 2006

46. Pötgens AJ, Lubsen NH, van Altena MC, Schoenmakers JG, Ruiter DJ and de Waal RM: Vascular permeability factor expression influences tumor angiogenesis in human melanoma lines xenografted to nude mice. Am J Pathol 146197-146209, 1995. 